\title{
Phase Space Solutions in Scalar-Tensor Cosmological Models
}

\author{
José C. C. de Souza
}

Departamento de Física Matemática, Instituto de Física, Universidade de São Paulo, C.P. 66318, 05315-970 São Paulo, SP, Brazil

and Alberto Saa

Departamento de Matemática Aplicada, IMECC - UNICAMP, C.P. 6065, 13083-859 Campinas, SP, Brazil

(Received on 15 October, 2005)

\begin{abstract}
An analysis of the solutions for the field equations of generalized scalar-tensor theories of gravitation is performed through the study of the geometry of the phase space and the stability of the solutions, with special interest in the Brans-Dicke model. Particularly, we believe to be possible to find suitable forms of the BransDicke parameter $\omega$ and potential $V$ of the scalar field, using the dynamical systems approach, in such a way that they can be fitted in the present observed scenario of the Universe.
\end{abstract}

\section{SCALAR-TENSOR THEORIES OF GRAVITATION}

In a homogeneous and isotropic space, described by the Friedmann-Lematre-Robertson-Walker metric

$$
\mathrm{d} s^{2}=-\mathrm{d} t^{2}+a^{2}(t)\left[\frac{\mathrm{d} r^{2}}{1-K r^{2}}+r^{2}\left(\mathrm{~d} \theta^{2}+\sin ^{2} \theta \mathrm{d} \varphi^{2}\right)\right],
$$

where $a$ is the scale factor and $K$ is the spatial curvature index, gravitation can be described by an action of the kind

$$
S=\frac{1}{16 \pi} \int \mathrm{d}^{4} \sqrt{-g}\left[\phi R-\frac{\omega(\phi)}{\phi} g^{a b} \nabla_{a} \phi \nabla_{b} \phi-V(\phi)\right]+S^{m},
$$

where $S^{m}$ is the action of usual matter, $g$ is the determinant of the metric tensor, $\omega$ is a coupling function (which we will eventually assume to be a constant, known as Brans-Dicke parameter) and $V(\phi)$ is the potential of the scalar field $\phi$ [2].

From (2), we obtain for the field equations:

$$
\begin{gathered}
H^{2}=-H\left(\frac{\dot{\phi}}{\phi}\right)+\frac{\omega}{6}\left(\frac{\dot{\phi}}{\phi}\right)^{2}+\frac{V(\phi)}{6 \phi}-\frac{K}{a^{2}}+\frac{8 \pi \rho^{m}}{3 \phi}, \\
\dot{H}=-\frac{\omega}{2}\left(\frac{\dot{\phi}}{\phi}\right)^{2}+2 H\left(\frac{\dot{\phi}}{\phi}\right) \\
+\frac{1}{2(2 \omega+3) \phi}\left[\phi \frac{\mathrm{d} V}{\mathrm{~d} \phi}-2 V+\frac{\mathrm{d} \omega}{\mathrm{d} \phi}(\dot{\phi})^{2}\right] \\
+\frac{K}{a^{2}}-\frac{8 \pi}{(2 \omega+3) \phi}\left[(\omega+2) \rho^{m}+\omega P^{m}\right], \\
\ddot{\phi}+\left(3 H+\frac{1}{2 \omega+3} \frac{\mathrm{d} \omega}{\mathrm{d} \phi}\right) \dot{\phi}=\frac{1}{2 \omega+3}\left[2 V-\phi \frac{\mathrm{d} V}{\mathrm{~d} \phi}+\right. \\
\left.8 \pi\left(\rho^{m}-3 P^{m}\right)\right],
\end{gathered}
$$

where $H \equiv \dot{a} / a$ is the Hubble parameter and $\rho^{m}$ and $P^{m}$ are the energy density and the pressure of the material fluid.

As usual, we parameterize the equation of state for the fluid as $P^{m}=(\gamma-1) \rho^{m}$ with $\gamma$ a constant chosen to indicate a variety of fluids that are predominantly responsible for the energy density of the Universe. We can see that through the energy conservation equation $\dot{\rho}^{m}+3 H\left(\rho^{m}+P^{m}\right)=0$ we obtain $\rho^{m}=\rho_{0} / a^{3 \gamma}$, with $\rho_{0}$ a constant.

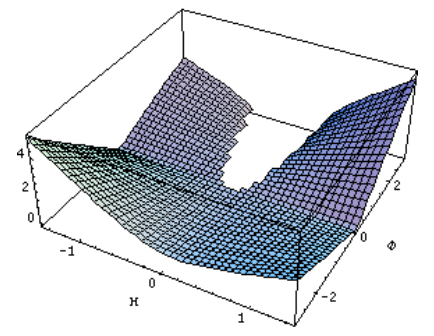

FIG. 1: Upper sheet of the of the phase space for a model with $\omega=10$ (Brans-Dicke), corresponding to the positive sign in eq. (7) [2]. The "hole" in the surface indicates the region forbidden for the orbits of the solutions.

\section{THE CASE FOR $V=\frac{1}{2} m^{2} \phi^{2}$ AND $K=0$}

In the referred paper [2], the author proceeds to show the phase space allowed for the orbits of solutions for these field equations in several cases with different potentials and parameters $\omega$. For example, in the case of vacuum, flat space $(K=0)$ and potential $V=\frac{1}{2} m^{2} \phi^{2}$, equation (3) was rearranged as (making $m \equiv 1$ )

$$
\omega \dot{\phi}^{2}-6 H \phi \dot{\phi}+\left(\frac{1}{2} \phi^{2}-6 H^{2} \phi\right) \phi=0
$$

which has the solutions

$$
\dot{\phi}_{ \pm}(H, \phi)=\frac{1}{\omega}\left[3 H \phi \pm \sqrt{3(2 \omega+3) H^{2} \phi^{2}-\frac{1}{2} \omega \phi^{2}}\right] .
$$

The assumption of flat space is required in order to reduce the dimensionality of the phase space.

We want to analyze qualitatively the geometry of the phase space $(H, \phi, \dot{\phi})$, expecting to infer the form of the functions $\omega(\phi)$ and $V(\phi)$ to fit better the available data on the structure of the Universe.

The phase space for this situation is composed of a 2-d surface with two sheets, related to the lower and upper signs in eq. (7). Figures 1-3 show the phase space for the choice $\omega=10$.

The fixed points for this dynamical system, obtained making $\dot{H}=\dot{\phi}=0$, are de Sitter solutions, given by $H_{0}=$ $\pm \sqrt{\phi_{0} / 12}$, with constant $H_{0}$ and $\phi_{0}$. 


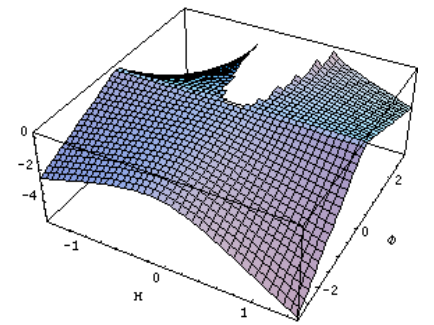

FIG. 2: Lower sheet of the phase space, now corresponding to the negative sign in eq. (7).

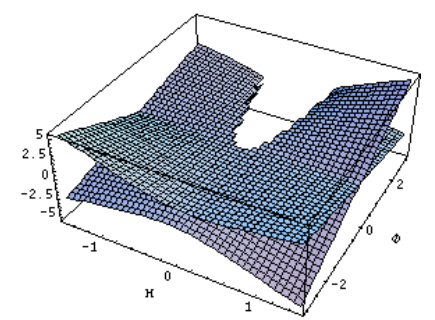

FIG. 3: The complete phase space composed of the upper and lower sheets linked to each other at the boundary of the forbidden region.

\section{THE CASE FOR $V=\Lambda \phi$ AND $K=0$}

Following other works ([3]-[5]) which give a complete analysis of the phase space for Brans-Dicke model with a cosmological constant $\Lambda$ (simply making $V(\phi)=\Lambda \phi$ in the action), we can illustrate the situation in which $\omega$ has a very large value and $\gamma=0$. Therefore, the energy density of the fluid is a constant $\rho_{0}$. It should be emphasized that recent observational and simulation results seem to favor a scenario very similar to this one ([6],[11]-[14]). The solutions in this case are written as

$$
\dot{\phi}_{ \pm}(H, \phi)=\frac{1}{\omega}\left[3 H \phi \pm \sqrt{9 H^{2} \phi^{2}-\omega\left[\phi^{2}\left(\Lambda-6 H^{2}\right)+16 \pi \rho_{0}\right]}\right] .
$$

With this solutions, we are able to show the phase space for a particular choice of constants $\Lambda, \omega$ and $\rho_{0}$.

We proceed to find the dynamical equations system for this simple model, as done before.

Naming $\Delta$ the expression under the root in eq.(8), we can write the equation for $\dot{H}$ :

$$
\begin{aligned}
\dot{H}_{ \pm}= & -\frac{1}{2 \omega \phi^{2}}[3 H \phi \pm \sqrt{\Delta}]^{2}+\frac{2 H}{\omega}\left[3 H \pm \frac{\sqrt{\Delta}}{\phi}\right] \\
& -\frac{1}{2(2 \omega+3)}\left(\frac{\Lambda}{2}+\frac{16 \pi \rho_{0}}{\phi}\right) .
\end{aligned}
$$

Now, equations (8) and (9) form the system for which the fixed points are the solutions $H_{0}= \pm \sqrt{8 \pi \rho_{0} / 3 \phi_{0}^{2}+\Lambda / 6}$.

Of special interest is the search for the most adequate functions $\omega(\phi)$ and $V(\phi)$, that may be more complicated than what was assumed until here.

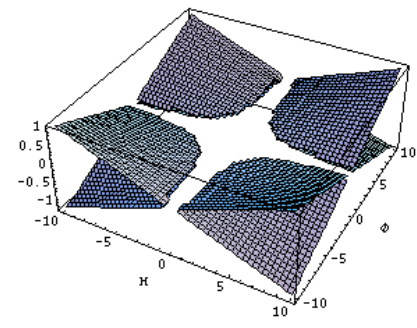

FIG. 4: Complete phase space for a Brans-Dicke model with a cosmological constant $\Lambda=1$, energy density $\rho_{0}=2$ and constant parameter $\omega=50000$, showing two sheets linked by the boundary of the forbidden region, as in the precedent case.

\section{CONCLUSIONS}

The method of analyzing the geometry of the phase space have proved to be a useful tool in the search for the solutions of the field equations of generalized gravity models. Our aim is to achieve a complete analysis of the simple model presented before (including the stability of the solutions, via Lyapunov's direct method [1], in order to investigate further its attraction basin) and to apply more sophisticated functions to it.

\section{ACKNOWLEDGMENTS}

This work is supported by Conselho Nacional de Desenvolvimento Científico e Tecnológico (CNPq). We would like to thank F. C. Carvalho for useful discussions.
[1] J. LaSalle and S. Lefschetz, Stability by Lyapunov's Direct Method. Academic Press (1967)

[2] V. Faraoni, Annals of Physics 317, 366 (2005)
[3] S. J. Kolitch, Annals of Physics 246, 121 (1996)

[4] S. J. Kolitch, Annals of Physics 241, 128 (1995)

[5] C. Santos and R. Gregory, Annals of Physics 258, 111 (1997) 
[6] A. G. Sanchez et al., astro-ph/0507583

[7] G. Esposito-Farse and D. Polarski, Phys. Rev. D63, 063504 (2001)

[8] A. Saa et al., Phys. Rev. D63 067301 (2001); Int. J. Theor. Phys. 40, 2295 (2001); L.R. Abramo, L. Brenig, E. Gunzig, and A. Saa, Phys. Rev. D 67, 027301 (2003); gr-qc/0305008.

[9] J. D. Barrow and J. P. Mimoso, Phys. Rev. D 50(6), 3746 (1994)

[10] F. C. Carvalho and A. Saa, Phys. Rev. D70, 087302 (2004)
[11] G. Esposito-Farse, gr-qc/0409081

[12] B. Bertotti, L. Iess, and P. Tortora, Nature 425, 374 (2003)

[13] V. Acquaviva, C. Baccigalupi, S. M. Leach, Andrew R. Liddle, and F. Perrotta, astro-ph/0412052

[14] A. R. Liddle, A. Mazumdar, and J. D. Barrow, Phys. Rev. D 58 , 027302 (1998) 